\title{
Parámetros nutricionales para desarrollar un buen entrenamiento físico y calidad de vida en el deporte
}

Nutritional parameters to develop a good physical training and quality of life in sport

Efraín Velasteguí López. ${ }^{1}$ Lic. Joel García Espinosa. ${ }^{2}$ \& Est. Liana Borges Sierra. ${ }^{3}$

\begin{abstract}
.
The nutrition is one of the most important factors in the realization of the physical exercise, it is the guarantee of its good execution. However, the importance is not given that corresponds him for its magnitude. I motivate that incentivated the realization of this work with the objectives of to determine the paper of the nutrition in the physical exercise and to identify the proportions of nutrients adapted in the sport diet. After an exhaustive revision it was determined that the diet is a fundamental element and with an indissoluble relationship with the exercise.
\end{abstract}

\section{Resumen.}

La nutrición es uno de los factores más importantes en la realización del ejercicio físico, es la garantía de su buena ejecución. Sin embargo, no se le es dada la importancia que le corresponde por su magnitud. Motivo que incentivó la realización de este trabajo con los objetivos de determinar el papel de la nutrición en el ejercicio físico e identificar las proporciones de nutrientes adecuados en la dieta deportiva. Después de una exhaustiva revisión se determinó que la dieta es un elemento fundamental y con una relación indisoluble con el ejercicio. Dieta que no

1 Ciencia digital, Ambato, Ecuador, luisefrainvelastegui@cienciadigital.org https://orcid.org/0000-0002-7351-0105

${ }^{2}$ Ciencias Médicas Mayabeque, Mayabeque, Cuba, jgarcia@infomed.sld.cu iD https://orcid.org/0000-00027351-0106

${ }^{3}$ Ciencias Médicas Mayabeque, Mayabeque, Cuba, lborgues@infomed.sld.cu iD https://orcid.org/0000-00027351-0107 
Diet that should not be substituted by supplements vitamínicos, but that passed two hours after the exercise it should be eaten abundant carbohydrates to recover the wornout energy. Care, it should not exclude of their diet the other nutrients all they are necessary, even the water.

Keywords: Nutrition, physical exercise debe ser sustituida por suplementos vitamínicos, pero que pasadas dos horas después del ejercicio se debe comer abundantes carbohidratos para recuperar la energía gastada. Cuidado, no debe excluir de su dieta los otros nutrientes todos son necesarios, incluso el agua.

Palabras claves: Nutrición, ejercicio físico.

\section{Introducción.}

La nutrición es un tema que cada día cobra más fuerza en el mundo de la actividad física. Es éste un aspecto importante a considerar cuando se quiere obtener el máximo rendimiento en la actividad física. Pero... ¿Qué es la Nutrición? La nutrición no es más que la ingesta de alimentos en relación con las necesidades dietéticas del organismo.

Una buena dieta equilibrada y combinada con el ejercicio físico regular, es un elemento fundamental para la buena salud. Sin embargo, la alimentación debe ser adecuada tanto en calidad como en cantidad, porque debe reponer la energía gastada durante la actividad física para evitar la aparición de un estado posterior de agotamiento. El aporte de carbohidratos, proteínas, grasas, minerales y vitaminas (o sea nutrientes, aunque también es un nutriente el agua) debe ser proporcional a la actividad física que se realiza y a las características físicas y patológicas de la persona.

Los alimentos son aquellas sustancias de nuestro entorno que seleccionamos para comerlas. La cantidad y el tipo de alimentos que una persona consume diariamente, se denomina dieta alimenticia. El valor nutricional de la dieta depende de los alimentos que tomemos. Ellos se agrupan teniendo en cuenta su composición, en base a este criterio se constituyen siete grupos de alimentos:

1. Leche y sus derivados: proteínas y minerales.

2. Carnes, pescados y huevos: proteínas.

3. Patatas, legumbres y frutos secos: carbohidratos y proteínas.

4. Hortaliza y verduras: vitaminas y minerales.

5. Frutas: vitaminas, minerales y carbohidratos.

6. Cereales, azúcares y derivados: carbohidratos. 
7. Aceites y grasas: grasas.

Los nutrientes encargados de proveer al organismo de energía son los carbohidratos mayormente. Las proteínas sólo se emplean energéticamente en situaciones muy especiales, como en estado de inanición (debilidad grande por falta de alimento).

La calidad de vida puede mejorarse considerablemente mediante el ejercicio físico y un aporte nutricional correcto. Una buena alimentación forma parte del llamado entrenamiento invisible e influye de manera decisiva, tanto en el incremento de la resistencia física como en facilitar una mejor recuperación. Siendo esa nuestra motivación para investigar sobre el tema, con los objetivos de: determinar el papel de la nutrición en el ejercicio físico e identificar las proporciones de nutrientes adecuados en la dieta deportiva.

\section{Desarrollo}

El ejercicio físico es necesario para el correcto funcionamiento de cuerpo y la mente. Una buena combinación de dieta y deporte mejorará nuestras capacidades físicas, nos ayudará a combatir el estrés, la ansiedad y contribuirá a perder peso, en caso de que así se precise.

En el rendimiento de una persona activa influyen diversos factores, como el entrenamiento, la genética y la alimentación. Éste último es un factor muy importante a la hora de mejorar el éxito en un deporte, hasta tal punto que el tiempo de entrenamiento y de preparación, pueden verse afectados por una incorrecta alimentación. La dieta de cada persona debe cubrir sus demandas energéticas en pos de las actividades que realiza para mantener un peso corporal adecuado. Por ejemplo, una persona sedentaria debe ingerir solamente la cantidad de calorías suficiente para poder realizar sus actividades diarias. Pero las actividades físicas intensas suponen grandes demandas y la persona muy activa necesita un alto aporte de nutrientes que permita cubrir el gran gasto de energía que se produce a nivel muscular.

En este punto es importante aclarar que, entre la comida y el inicio de la actividad física, deben pasar al menos de 2 a 21/2 horas. No es recomendable comer grandes cantidades de carbohidratos ni de alimentos que necesiten largos periodos de tiempo para ser digeridos (ej. carnes grasas), también deben evitarse las bebidas gaseosas, la cafeína y el alcohol. Los alimentos hervidos son los más preferibles.

En un segundo momento, la nutrición después del ejercicio, ha de ayudar al organismo a reponerse del esfuerzo realizado. Para ello, es preciso rehidratar el organismo y reponer las reservas de carbohidratos y proteínas. La mayor parte de la actividad metabólica que se produce en el cuerpo en esta fase se centra en dos aspectos, la reparación de las fibras musculares dañadas y la eliminación de las sustancias de desecho producidas durante el ejercicio. Si el entrenamiento ha provocado una gran pérdida de líquido a través del sudor, es necesario reponerlo tomando bebidas con glucosa, que ayudan a restablecer las reservas 
de carbohidratos. Han de pasar entre 30 y 60 minutos antes de tomar alimentos sólidos. Alimentos como los huevos, la leche, la levadura de cerveza, el pescado, la carne y el hígado son adecuados para contribuir a una mejor recuperación.

La comida posterior a la actividad física cotidiana será normal, según el apetito de la persona activa. Sin embargo, ha de cuidarse especialmente, si la actividad física ha requerido un esfuerzo intenso y prolongado. Entonces se aconseja aumentar la ingestión de carbohidratos y reducir la de grasas animales durante 2 o 3 días -en esta comida-.

Descuidar la nutrición en calidad, cantidad y tiempo correcto tras el ejercicio, supone un rendimiento energético bajo, acompañado de resultados deficientes tanto en la actividad física como en la recuperación.

La proporción y cantidad de los alimentos que comemos determinan si vamos a tener en buena salud o vamos a desarrollar patologías nutricionales. El consumo excesivo y desproporcionado de algunos alimentos puede crear desequilibrios que eventualmente destruyen nuestra salud y fomentan: la obesidad, problemas cardiovasculares, diabetes y otros trastornos. Los vegetarianos estrictos (veganos) que no comen productos de origen animal o productos lácteos tienen un alto riesgo de desnutrición, especialmente durante el embarazo o lactancia. La ingesta inadecuada de proteínas provoca una forma de malnutrición llamada kwashiorkor. Niveles bajos de minerales o vitaminas pueden causar enfermedades como la anemia, bocio, pelagra, raquitismo, escorbuto, y retraso mental. Mientras que la ingesta diaria de vitaminas y minerales o alimentos fortificados, ha reducido considerablemente la incidencia de muchas enfermedades de deficiencia en la población general; pero las vitaminas y minerales, al igual que todos los componentes de nuestra dieta, deben tomarse en proporciones adecuadas. Los niveles bajos de vitaminas son malos, pero los niveles altos también pueden ser peligrosos. La vitamina A, por ejemplo, es necesaria para el mantenimiento de los ojos y la piel, pero en altas concentraciones es tóxica y puede causar defectos congénitos en el feto. La variedad, el equilibrio y la moderación son muy importantes en la nutrición. De ahí determinamos, que comer bien ayuda a mejorar la salud, resistencia y energía.

La buena nutrición es especialmente importante para los deportistas que desean optimizar sus rendimientos; esto es aplicable no sólo a los deportistas profesionales y de élite, sino también a los deportistas de ocio. Incluso con una correcta combinación de genética, preparación física y entrenamiento, los deportistas mal alimentados son incapaces de desarrollar por completo su potencial, tanto si es para correr 5 millas durante la hora de la comida o hacer una clase de aeróbico después del trabajo.

Según la nutrióloga deportiva Nancy Clark: "Los deportistas, tanto en deportes de intensidades cortas como en deportes de resistencia, obtienen mejores rendimientos con una dieta rica en carbohidratos y baja en proteínas". 
Esto es comprensible ya que los hidratos de carbono son la fuente más importante de energía durante la actividad física intensa. Ellos se presentan en dos formas: simples y complejos. Los simples se encuentran en las frutas, zumos, leche, yogures y dulces; mientras que los complejos se encuentran en los granos completos, verduras, pastas, arroz y pan. El cuerpo metaboliza ambas formas en glucosa para las necesidades energéticas inmediatas. Los excesos de glucosa se almacenan como glucógeno fundamentalmente en los músculos y en el hígado, para carburante (mezcla de hidrocarburos que se emplea en los motores de explosión y de combustión interna) del ejercicio. A pesar de ser los nutrientes más importantes, son los que menos se almacenan en el cuerpo. Tras 2 horas de ejercicio u 8 horas de paso rápido se pueden vaciar significativamente sus almacenamientos. En los deportistas, este vaciamiento puede ocasionar fatiga y descenso del rendimiento. Además, para seguir una dieta rica en hidratos de carbono como parte del régimen de salud, se deben consumir después del entrenamiento, ya que maximiza la recuperación.

El consumo de carbohidratos en la comida (después del ejercicio) debe incrementarse desde la cantidad normal de 350 - 600 gramos. Un consumo que exceda de los 600 gramos no proporcionará concentraciones mayores de glucógeno muscular. Los hidratos de carbono deberán ser complejos, ya que proporcionan más concentración de glucógeno que los simples. Cuando la mayor parte de los deportistas piensa en carbohidratos, automáticamente los asocia con pastas, cereales o granos, sin darse cuenta de que se encuentran en las frutas y son los nutrientes principales de la mayoría de las verduras.

En el caso de las proteínas, son necesarias para construir y reparar los músculos, ligamentos, tendones y otros tejidos. No se utilizan como fuente especial de energía. Menos del 10\% de la energía utilizada durante el entrenamiento deriva del metabolismo proteico. Los deportistas necesitan solamente entre 1 y 2 gramos de proteínas por kilo de peso corporal al día. Sólo una cantidad limitada de proteínas es necesaria para la reconstrucción tisular. Su exceso se convierte en grasa. Por lo que una pequeña ración de alimentos ricos en proteínas en cada comida es suficiente para mantener una actividad deportiva vigorosa y cumplir con los requerimientos corporales básicos.

La grasa es la fuente de energía más concentrada de los nutrientes diarios. Se clasifican en saturadas y no saturadas (poliinsaturadas y monoinsaturadas). Los ácidos grasos saturados (grasa de la carne de vaca, cerdo, cordero y aves; productos lácteos, aceite de coco, de palma hidrogenado y chocolate) tienden a aumentar los niveles de colesterol en la sangre. Las grasas saturadas deben representar no más del $10 \%$ de la ingesta calórica diaria. En cambio, las grasas poliinsaturadas derivan principalmente de los aceites vegetales (trigo, semilla de algodón, soja y girasol) y de los aceites de pescado. Las grasas monoinsaturadas se encuentran en el aguacate, y en los aceites de oliva, cacahuete y frutos secos. Las grasas no saturadas son las más recomendables y sanas. Sin embargo, los alimentos consumidos antes de hacer ejercicio deben ser bajos en grasas, debido a que éstas alargan el proceso de la 
digestión, enlentecen el vaciamiento del estómago y del tracto gastrointestinal superior; además pueden dificultar la respiración, la circulación y eventualmente producir náuseas y vómitos. En la vida todo tiene un equilibrio y cuando esta falta, ya sea por exceso o defecto siempre es para mal, en la salud; y consumir una cantidad insuficiente de grasas crea problemas para el deportista, en particular la inadecuada ingesta calórica. Es apropiada una cierta cantidad de grasa en cada comida, pero evitando excesivas cantidades de fritos, comidas grasientas, aceitosa o con mantequillas, las cuales llenarán el estómago, pero dejarán a los músculos sin carburante.

Las vitaminas y minerales son importantes para muchas reacciones metabólicas, pero no proporcionan energía. Es un error muy difundido entre los deportistas que los suplementos vitamínicos mejorarán los rendimientos deportivos. Muchos de ellos consumen grandes cantidades de suplementos vitamínico-minerales en la creencia de que estas dosis mejorarán su rendimiento; sin embargo, la ciencia médica no ofrece evidencia alguna de que éste mejore con el consumo de tales suplementos. El hombre sano no requiere suplementos vitamínicominerales en tanto que esté digiriendo una dieta variada. Los estudios más recientes revelan que, debido a los cambios de los hábitos dietéticos de la población (el incremento del consumo de comidas procesadas y "comidas rápidas"), puede haber mucha gente cuya ingesta vitamínico-mineral sea insuficiente. Sin embargo, se indica a estos individuos a mejorar su dieta en lugar de recurrir a suplementos.

Cuando los deportistas utilizan suplementos nunca deben exceder del 150\% de la dosis diaria recomendada. Aun cuando la mayor parte de los nutriólogos aseveran que no se producirá ningún daño por tomar estas cantidades, también establecen que no existen evidencias de que esta práctica sea beneficiosa. Consumir grandes dosis de vitaminas es una práctica popular en aumento entre los deportistas, es costoso y genera falsas expectativas. Una de las mayores preocupaciones de los profesionales médicos es la evidencia de que el consumo masivo de vitaminas y minerales puede ser tóxico. Por lo que consideramos que el alto rendimiento sólo es posible manteniendo los almacenamientos adecuados de carbohidratos, lo cual se consigue comiendo siempre de manera adecuada. Sin embargo, conseguir estos depósitos exige planificar la ingesta de inmediatamente antes y después del ejercicio y, en ocasiones, durante la propia actividad.

Por lo que determinamos que los requerimientos nutricionales pueden satisfacerse suficientemente con una dieta básica, equilibrada y no mediante comidas mágicas, dietas maravillosas o suplementos dietéticos que pueden resultar, incluso, perjudiciales. Aunque los suplementos son legales, estas sustancias pueden causar efectos secundarios y sólo deben ser utilizadas bajo supervisión médica, pues en muchos casos los problemas surgen cuando se abusa de ellas o cuando no se utilizan adecuadamente (por ejemplo, si se reemplazan por una buena alimentación). Entre las sustancias más conocidas están: 
- Creatina: es uno de los suplementos más populares. En estado natural se encuentra en la carne roja, y también es producida por el cuerpo a partir de algunos aminoácidos. $\mathrm{Su}$ función consiste en ayudar a convertir el ADP (adenosin difosfato) en ATP (adenosin trifosfato), que es el combustible que utilizan los músculos para realizar la contracción muscular.

- L-Carnitina: también conocida como vitamina Bt, es una sustancia natural presente en el cuerpo humano. La utiliza para transportar ácidos grasos de cadena larga hasta las mitocondrias de las células, donde son quemados para obtener energía.

- L-Glutamina: es un aminoácido que nuestro cuerpo puede sintetizar. Interviene en la formación del glucógeno y ayuda a reponer sus reservas después de actividades intensas. Se encuentra en lácteos, en carnes, en los cacahuetes y en las almendras.

Por último, se debe destacar, que también se consumen productos ilegales: anabolizantes, esteroides, hormonas... Evidentemente están absolutamente prohibidos y son muy perjudiciales para la salud. Tan perjudiciales para la salud como una mala nutrición. Si, una mala nutrición puede reducir la inmunidad, aumentar la vulnerabilidad a las enfermedades, alterar el desarrollo físico - mental y reducir la productividad. Pero esto no se queda aquí ¿Sabías que los fluidos son parte de una dieta saludable? Pues así es, sin embargo, la hidratación adecuada, es la ayuda que más frecuentemente se ignora para el rendimiento deportivo.

Beber más líquido no solo ayuda a prevenir las deshidrataciones sino también el sobrecalentamiento. Para los deportistas ocasionales, el agua es suficiente como fluido de reemplazamiento. Si el ejercicio dura más de 30 minutos (especialmente en alta temperatura o humedad), el participante debe beber un vaso de agua fría cada 20 minutos como máximo. Los deportistas no deben esperar a beber hasta que tengan sed, ya que esta sensación aparece posteriormente a las necesidades reales.

Para actividades de resistencia que duran más de 2 horas, el rendimiento deportivo no sólo depende del balance hídrico, sino también de los niveles de glucosa en sangre. Para estos casos, muchas bebidas deportivas proporcionan agua y un suplemento de pequeñas cantidades de azúcar y sodio. Estudios actuales sugieren que los deportistas de resistencia se benefician cuando consumen esta clase de bebidas comparadas con agua sola.

Después del ejercicio, la rehidratación se produce con mayor rapidez cuando el líquido que se ingiere contiene pequeñas cantidades de sodio. Las bebidas para el deporte proporcionan una pequeña cantidad de este mineral con este propósito.

Para finalizar es muy importante mencionar que las principales causas de la muerte prematura son las enfermedades del corazón, el cáncer, derrame cerebral y diabetes. El riesgo de estas 
enfermedades aumenta considerablemente con mala alimentación, el tabaquismo, el sobrepeso y la inactividad física. Usted tiene la responsabilidad de seleccionar buenos alimentos y cambiar el estilo de su vida para conseguir un futuro más saludable. Con buena salud y condición física le será más fácil lograr sus objetivos en la vida y ser feliz.

\section{Conclusiones.}

- La nutrición es fundamental para incrementar el rendimiento en el deportista y su recuperación. Esto se debe a que nutrientes como los carbohidratos son los que reponen la energía gastada a nivel muscular durante la actividad física. La proporción y cantidad de nutrientes en los alimentos que consumimos, determinarán nuestra salud. Nunca debe sustituirse una buena alimentación por suplementos vitamínicos minerales pues estos no reponen energía al organismo. En la dieta debemos incorporar proporciones adecuadas de cada nutriente, existiendo un equilibrio de estos en la alimentación, en ellos no puede haber ni exceso ni defecto porque ambos son perjudiciales. En la dieta deportiva es recomendable antes de hacer ejercicio comer alimentos hervidos y menos carbohidratos que retrasen la ingestión; y después de los ejercicios una dieta más rica en carbohidratos que en los otros nutrientes. No debe olvidarse que tanto los hidratos de carbono como los fluidos son indispensables para el deportista de élite o de ocio.

\section{Referencias bibliográficas.}

Dieta deportiva: nutrición para el ejercicio físico [Internet]. Madrid: Instituto Médico Láser; 1996 c 2017 [actualizado 2012 Jul 13; citado 2017 Sep 10]. Disponible en: https://www.iml.es/dieta-deportiva-nutricion-para-el-ejercicio-fisico/

El ejercicio físico es la clave para mejorar las recomendaciones [Internet]. Madrid: Muy interesante; 1995 c 2017 [actualizado 2016 marz 4; cited 2017 Sep 12]. Disponible en: https://www.google.com.cu/amp/a/www.muyinteresante.es/salud/articulo/elejercicio-fisico-es-clave-para-mejorar-las-recomendaciones-nutricionales901412075682/amp

La alimentación y la nutrición en el ejercicio físico [Internet]. Madrid: Portalfitness.com; 2000 c 2017 [actualizado 2013 dic 19; cited 2017 ago 30]. Disponible en: http://www.portalfitness.com/articulos.aspx

Nutrición tras el ejercicio físico [Internet]. Madrid: Sanitas; 1954 c 2017 [actualizado 2015 feb 13; citado 28 ago 2017]. Disponible en: http://www.sanitas.es/sanitas/seguros/es/particulares/biblioteca-de-salud/dietaalimentacion/deporte-alimentacion/san005132wr.html 
Relación entre la alimentación y el desarrollo de un ejercicio físico [Internet]. Madrid: FERTILAB; 1974 c 2017 [actualizado 2017 sep 17; cited 2017 Sep 12]. Disponible en:

http://www.fertilab.net/ginecopedia/ejercicios/aspectos_generales/relación_entre_la _alimentacion_y_desarrollo_de_un_ejercico_1 


\section{PARA CITAR EL ARTÍCULO INDEXADO.}

Velasteguí López, E., García Espinosa, J., \& Borges Sierra, L. (2019). Parámetros nutricionales para desarrollar un buen entrenamiento físico y calidad de vida en el deporte. Anatomía Digital, 2(4), 19-28. https://doi.org/10.33262/anatomiadigital.v2i4.1121

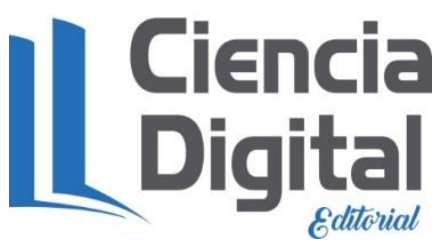

El artículo que se publica es de exclusiva responsabilidad de los autores y no necesariamente reflejan el pensamiento de la Revista Anatomía Digital.

El artículo queda en propiedad de la revista y, por tanto, su publicación parcial y/o total en otro medio tiene que ser autorizado por el director de la Revista Anatomía Digital.
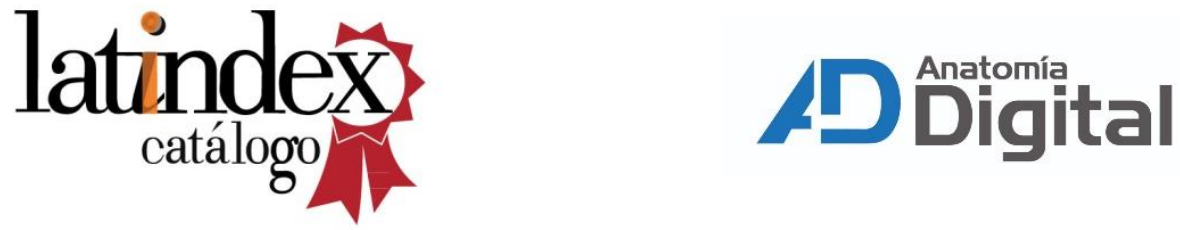